\title{
The use of computerised tomography in mental handicap patients
}

\author{
S. K. LEKH, Registrar in Psychiatry, Charing Cross and Westminster Rotation, \\ London; B. K. PuRI, Senior Registrar in Psychiatry, Charing Cross and Westminster \\ Rotation, London; and I. SINGH, Consultant Psychiatrist, Leavesden and Hillingdon \\ Hospitals
}

Since its inception (Hounsfield, 1973), computerised tomography (CT) has become an invaluable diagnostic and research tool, particularly in clinical neurology and neurosurgery. Clinically, CT has proved useful in differentiating between 'functional' and 'organic' psychiatric disorders where it is particularly helpful in the diagnosis of potentially treatable organic disorders. For example, Owens et al (1980) found clinically unsuspected intracranial pathology in 12 of 136 chronic schizophrenic patients examined by CT and Roberts \& Lishman (1984) found diagnosis, management, and/or prognosis were influenced in approximately $12 \%$ of cases referred by psychiatrists for CT imagining.

However, there is a paucity of evaluative studies of the use of CT in the field of mental handicap. In this study the current use of CT in a large mental handicap unit was examined.

\section{The study}

Leavesden mental handicap hospital serves the London boroughs of Hillingdon, Hammersmith, Harrow, Hounslow, Ealing, and Brent, a catchment population of one and a quarter million. The hospital population has been around 818 over the last four years. Data from in-patients and out-patients referred for CT scans, all to the CT Department of Northwick Park Hospital, over a three and a half year period (1987-1991) were examined.

Information collected from the medical records included demographic data, admission status and duration of admission, level of mental impairment, past and current medical history, past and current psychiatric history, mental state, neurological examination results, clinical diagnosis, the expected CT scan results, actual CT scan results, and clinical consequences of the CT findings.

Organic features were recorded as present (Roberts \& Lishman, 1984) if there was an episode of loss of, or impairment of consciousness, on two or more occasions; a confusional episode; excess alcohol intake plus one episode of hallucinations, or delirium tremens, or a convulsion, precipitated by alcohol withdrawal; impairment of memory; head injury with loss of consciousness and an associated period of amnesia; or a cerebral disorder (such as hemiparesis).

Organic features were also considered present if there was change in the pattern of neurological deficit; unexplained loss of skills; unexplained change in behaviour; memory impairment; or change in level of orientation.

Diagnosis were assigned according to the clinical features recorded in the medical records. The CT scan reports were categorised as showing no abnormality, equivocal change, or definite abnormality. The clinical consequences were considered under change in diagnosis and change in treatment.

\section{Findings}

A total of 29 CT investigations were performed or attempted. The number of patients involved was 26 , the investigation being carried out, or attempted, on two occasions for three of the patients.

The average age of the patients was 39.7 years, with a range of 17.1 to 72.9 . The male to female ratio was 15:14. The ratio of in-patients to out-patients was 22:7.

For the in-patients, the average length of admission at the time of referral was 19.0 years, with a range of one month to 51.0 years.

Using ICD-10 criteria for the level of mental handicap, the 29 cases were classified as borderline: 2 (7\%); mild: 9 (31\%); moderate: 9 (31\%); severe: 3 $(10 \%)$; profound: $5(17 \%)$; and not known: $1(3 \%)$. The aetiology was: genetic (Down's syndrome): 3 (10\%); genetic (phenylketonuria): $3(10 \%)$; fetal alcohol syndrome: 1 (3\%); birth trauma: $1(3 \%)$; postnatal infection: 1 (3\%); congenital-cause unknown: $17(59 \%)$; not known: 3 (10\%).

In 17 of the 29 cases there was no evidence of psychiatric disorder. Of those with psychiatric disorders there were drug-induced psychosis: $1(3 \%)$; alcoholic dementia: $1(3 \%)$; senile dementia: $1(3 \%)$; 
schizophrenia: 2 (7\%); psychotic, possibly schizophrenia: 2 (7\%); schizoaffective disorder: $1(3 \%)$; depressive illness: 3 (10\%); panic disorder: $1(3 \%)$; no psychiatric disorder: $17(59 \%)$.

In four cases there was no suggestion from the history of a cerebral lesion, while for 25 cases there was evidence of epilepsy: 15 (52\% of the 29 cases); two or more episodes of impairment/loss of consciousness: $3(10 \%)$; head injury \& loss of consciousness \& a period of amnesia: 7 (24\%); neurological disorder which may be caused by a cerebral lesion: $10(34 \%)$; cortical atrophy following alcohol abuse: $1(3 \%)$; nil: $4(14 \%)$. The total is greater than 29 because more than one class applied to one patient for some of the cases.

In ten cases changes found on neurological examination were indicative of a possible cerebral lesion, while in 19, no such changes were found. The detailed breakdown was motor changes: $8(28 \%)$; changes in peripheral reflexes: $2(7 \%)$; visual changes: $2(7 \%)$; the development of urinary incontinence: $2(7 \%)$; no changes: $19(66 \%)$.

In 18 cases, changes were found on mental state examination suggestive of a cerebral lesion: unexplained change in behaviour: $8(28 \%$ of the 29 cases); unexplained loss of skills: 5 (17\%); disorientation in time, place and person: $6(21 \%)$; memory impairment: 2 (7\%); fluctuating level of consciousness: 1 (3\%); no change in mental state: 11 $(38 \%)$.

The clinical diagnoses were: unspecified cerebral lesion: 17 (59\% of the 29 cases); cortical atrophy/ dementia: $9(31 \%)$; transient ischemic attack: $2(7 \%)$; hypopituitarism: 1 (3\%); aqueduct stenosis $\&$ ataxis (previously diagnosed): 1 (3\%); myocardial infarction: $1(3 \%)$; other: $2(7 \%)$. In some cases there was more than one diagnosis.

The CT investigations were expected by the referring consulting psychiatrists to confirm the absence of cerebral lesion in 15 cases, and to confirm its presence in 11 cases. In three cases the expectation was that the CT investigations would clarify the nature of the suspected lesion.

In eight cases definite abnormalities were found on CT investigation, while in 18 cases there was none found. The result was not available for one case and, in two cases, the patients were uncooperative.

In 13 cases the investigation confirmed the absence of a cerebral lesion. However, the expectations of the referring psychiatrists were found not to be fulfilled in six cases. In a 44 year old moderately handicapped epileptic female in-patient with increased frequency of fits, marked cerebellar atrophy and moderate cerebral atrophy were found, when the expectation had been that the investigation would show no abnormality. In four cases no abnormalities were found when expected, the working diagnoses having been hypopituitarism and transient ischaemic attacks; transient ischaemic and myocardial infarction; memory impairment because of hypoxia following attempted hanging; and senile dementia. In the sixth case, a 41 year old severely handicapped female in-patient with phenylketonuria, became increasingly aggressive, screaming, hitting out, and biting. $A$ clinical diagnosis of cerebral atrophy secondary to the phenylketonuria was made but no abnormality found.

The clinical consequences of the 26 CT investigations were then examined. In 21 cases there was a change in the diagnosis to a psychogenic cause in 13 cases; an organic cause in four cases; and from one organic cause to a different organic cause in one case. In the remaining eight cases the CT results did not lead to a change in diagnosis.

In terms of effect on treatment, in only one case did the CT result lead to a change. This was a patient for whom the CT investigation was requested because the referring psychiatrist suspected a subdural haematoma. The CT scan revealed a left subdural collection with shift of the lateral ventricles to the right and slight contralateral hydrocephalus. As a result a subdural evacuation was carried out.

\section{Comment}

This study was undertaken for two main reasons. First, we wished to carry out a clinical audit of the use of CT neuroimaging in mental handicap, to evaluate whether requests for this investigation were being made appropriately. The results suggest that the overall answer is in the affirmative. Indeed, not only do the results confirm its value in differentiating organic from functional disorders but (from the last mentioned case), it can be life-saving.

Second, the study examined whether CT neuroimaging has a role in mental handicap additional to its diagnostic function, and it was clear that the answer was again affirmative. In many families the parents react to their mentally handicapped child with delayed resolution of grief and guilt feelings. In such cases, once the psychiatrist has a tangible investigation results, such as a CT scan report, he/she may then be in a better position to discuss the existence of permanent brain damage with the parents who may the find it easier to come to terms with the handicap.

Another role for this investigation is in facilitating the performance of a complete assessment of a person with a severe learning difficulty for whom communication with others is exceedingly hard.

In conclusion, in the field of mental handicap, $\mathrm{X}$-ray computerised tomography is an under-used facility with the potential to make a valuable contribution. 


\section{References}

Hounsfield, G. N. (1973) Computerised transverse axial scanning (tomography): Part I. Description of a system. British Journal of Radiology, 46, 1016-1022.

OWEns, D. G. C., Johnstone, E. C., BYdDer, G. M. \& KREEL, L. (1980) Unsuspected organic disease in chronic schizophrenia demonstrated by computed tomography. Journal of Neurology. Neurosurgery and Psychiatry, 43, 1065-1069.

RoberTs, J. K. A. \& Lishman, W. A. (1984) The use of the CAT head scanner in clinical psychiatry. British Journal of Psychiatry, 145, 152-158.

\title{
Development of outcome measures in acute psychiatry
}

\author{
Femi Oyebode, Consultant Psychiatrist; Stuart Cumella, Senior Research Fellow, \\ Centre for Research and Information into Mental Disability; GILL GARDEN, Senior \\ Registrar; and JUDITH Nicholls, Senior Registrar, Queen Elizabeth Psychiatric \\ Hospital, Edgbaston, Birmingham B15 2QZ
}

This study is an evaluation of the use of two health status measures for monitoring the outcome of acute psychiatric treatment. The measures are the Sickness Impact Profile (SIP) and the Classification of Illness States (CIS). The SIP is a measure of the impact of sickness on patients' daily activities and behaviour. The CIS was initially developed to measure 'morbidity states' after hospital treatment and can be used to generate the 'Rosser Index', both of which have been shown to be valid and reliable measures (Rosser, 1988).

The aim of this investigation was to determine whether these health status measures were applicable in psychiatry. We also examined the ease of administering the questionnaires and whether sample survey of patients is practicable up to one year after discharge from hospital.

\section{The study}

All patients discharged from hospital in the adult psychiatric service between 1 April 1988 and 31 March 1989, resident at the time of their admission in two selected electoral wards of Birmingham, were eligible for inclusion. Two separate clinical teams were responsible for the wards which were selected to ensure inclusion of patients from a wide range of ethnic groups and domestic circumstances. Electoral ward ' $A$ ' had a population of 22,800 in 1981 and the Jarman index was 26.9. Electoral ward 'B' had a population of 24,400 in 1981 and the Jarman index was 1.5.
Patients who agreed to be interviewed were visited at home or in hospital or seen in out-patient clinics by a psychiatrist. Where there was no formal refusal to participate, a minimum of three visits were made to the home addresses before the patient was regarded as uncontactable. All patients who were seen completed the SIP, CIS and Hospital Anxiety and Depression (HAD) Scale. A short questionnaire with questions on type of housing, constitution of household, and employment circumstances was administered. Data on personal characteristics, diagnoses, and admission history of the total sample were obtained from the Unit Case Register. The interviews were conducted between April and September 1990.

\section{Findings}

One hundred and two patients were eligible for inclusion of whom 61 were contactable. Eleven patients refused to participate. Three were too cognitively impaired to complete the questionnaires and one patient had died. There was a lower response rate in Ward 'A' (18/51) compared with Ward 'B' $(29 / 51)$.

Of the 51 respondents, 38 were living in private households, six in hostels, and four in psychiatric hospital wards. In all, 29 patients had re-admissions in the year following the index discharge. Ten of the respondents were working at follow-up, one was in full-time education, and three were providing fulltime care for their families. The remainder included 